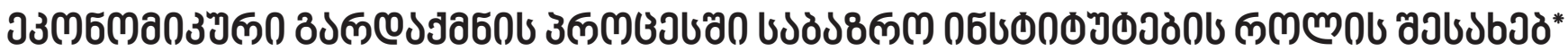

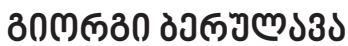

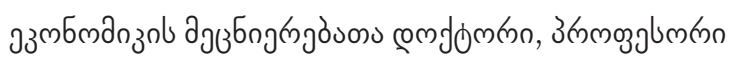

https://doi.org/10.35945/gb.2018.06.016

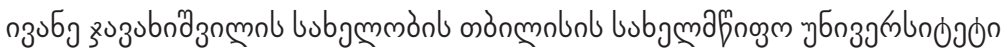

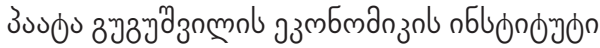

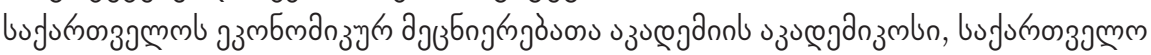

george.berulava@gmail.com

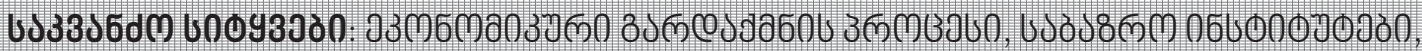

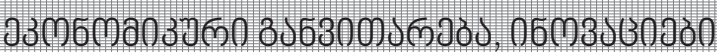

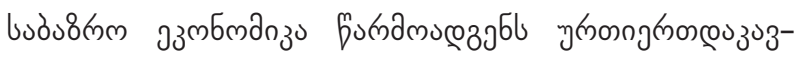

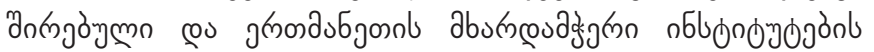

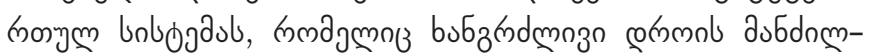

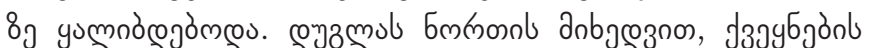

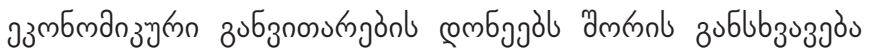

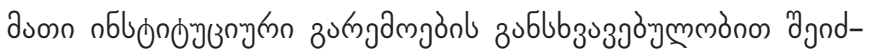

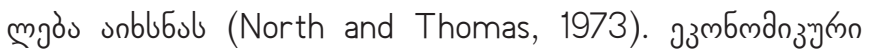

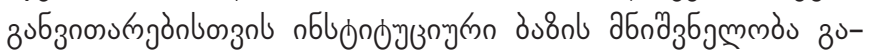

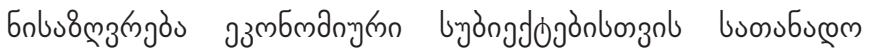

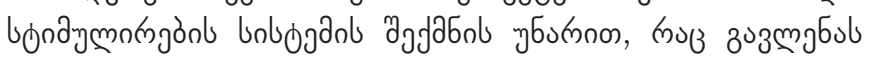

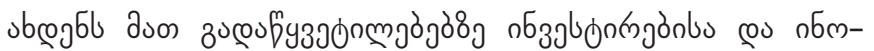

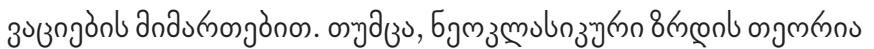

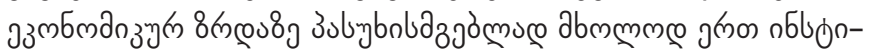

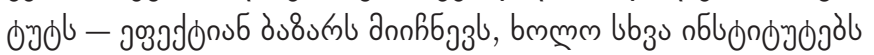

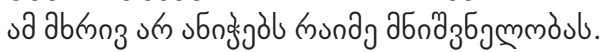

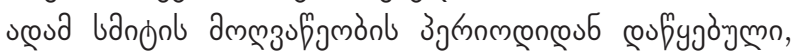

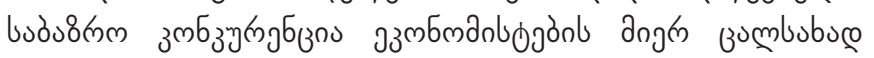

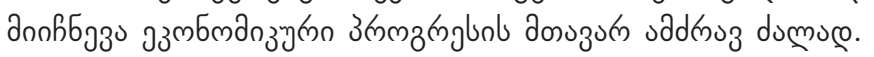

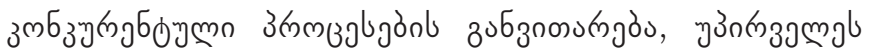

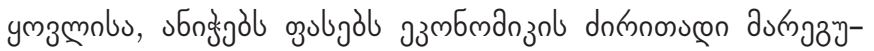

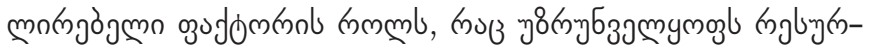

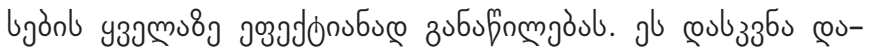
оुगd

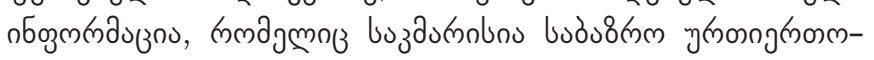

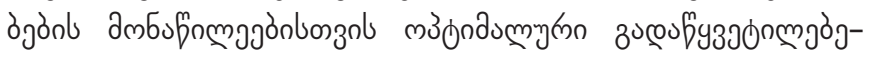

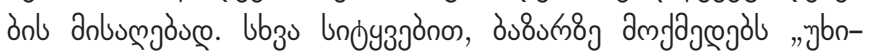

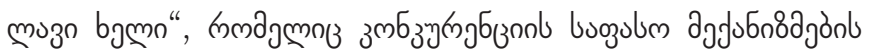

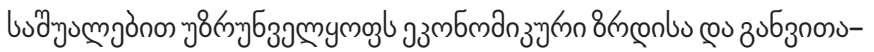

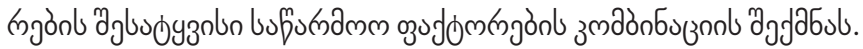

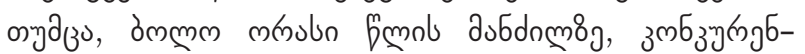

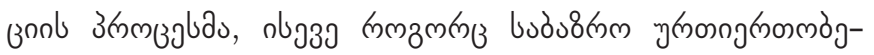

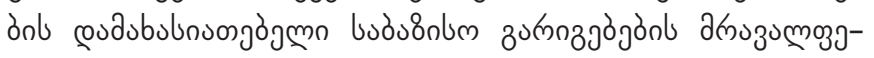

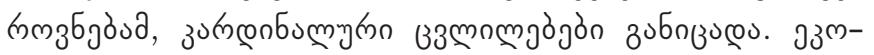

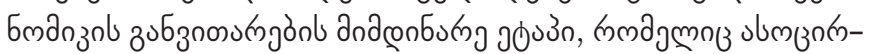

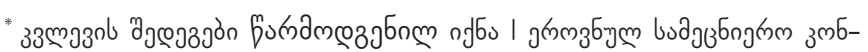

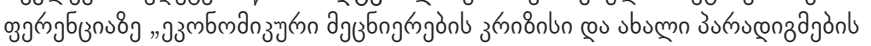
dngos"

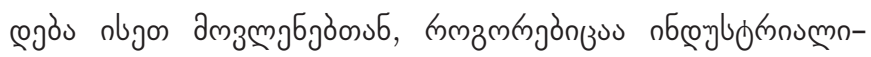

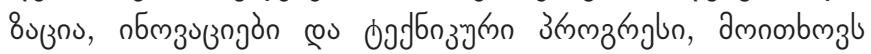

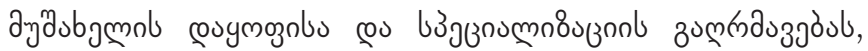

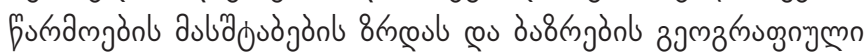

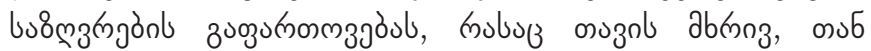

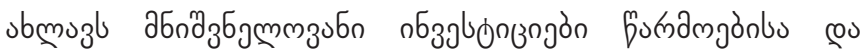

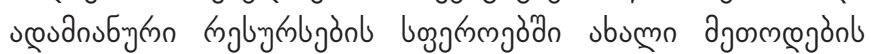

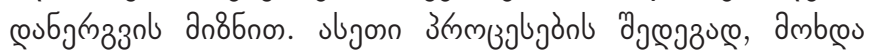

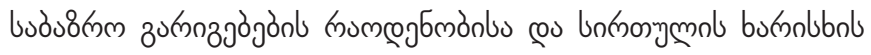

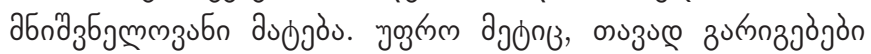

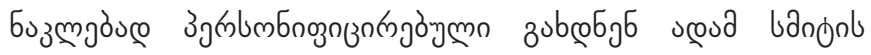

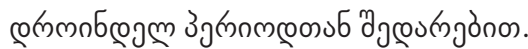

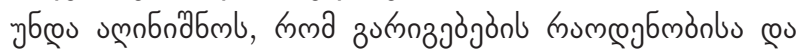

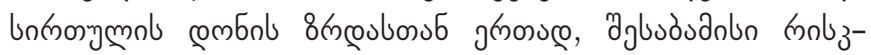

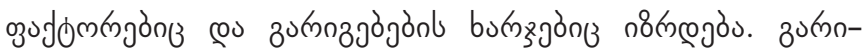

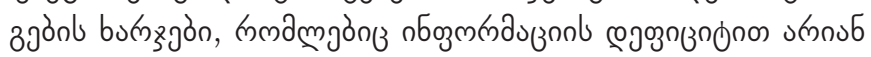

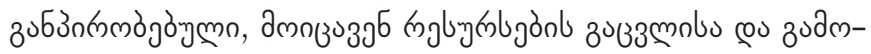

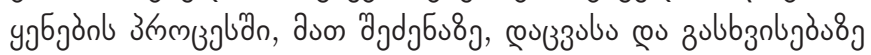

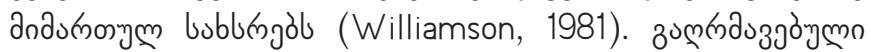

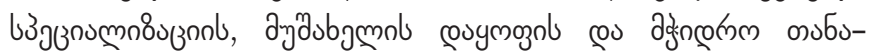

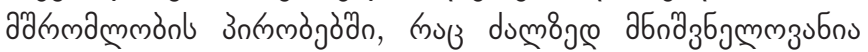

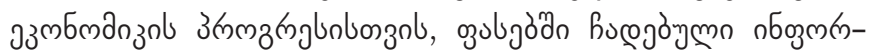

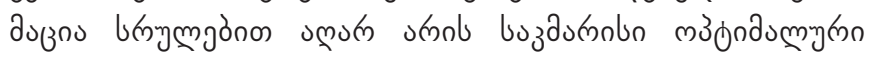

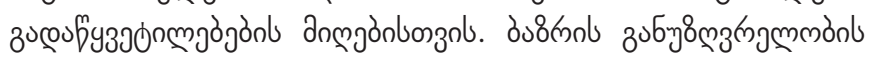

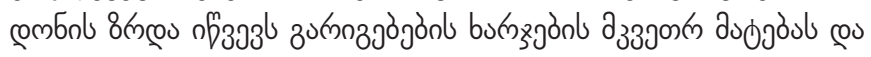

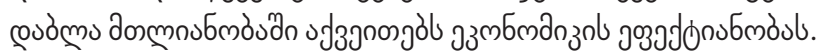

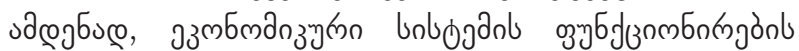

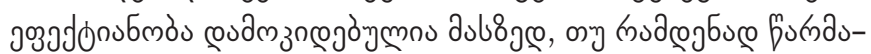

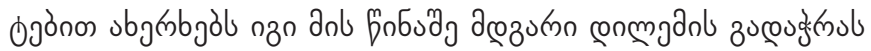

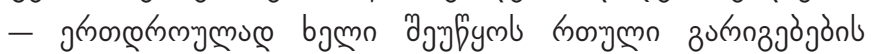

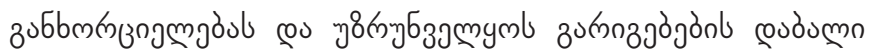

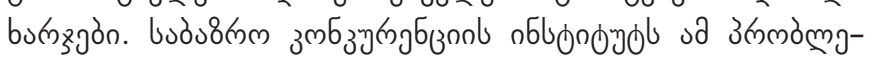

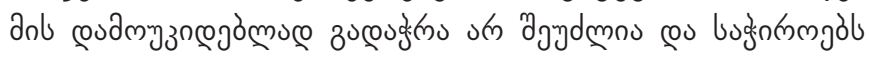

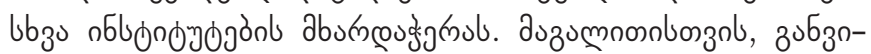

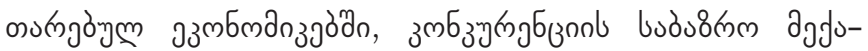

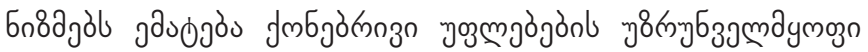

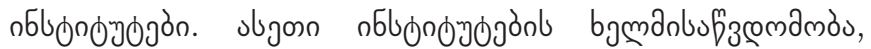




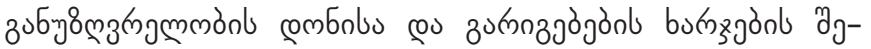

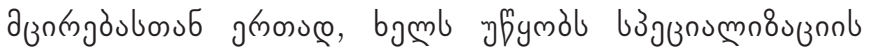
zuмnàs

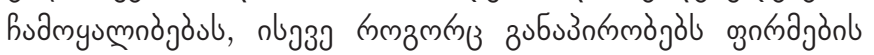

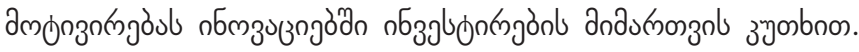

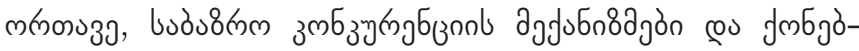

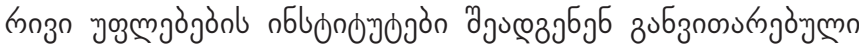

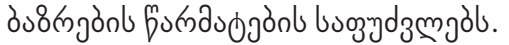

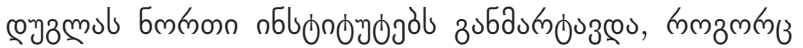

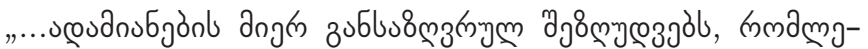

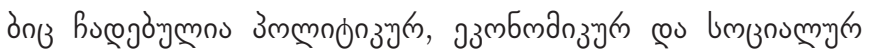

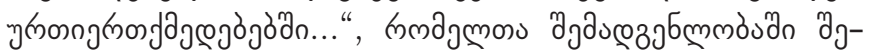

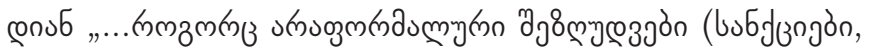

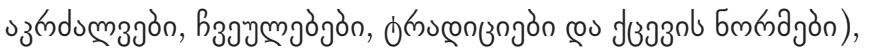

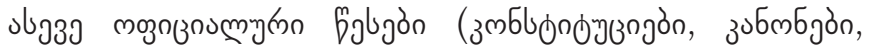

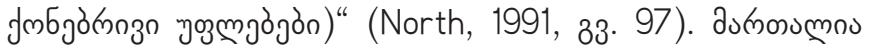

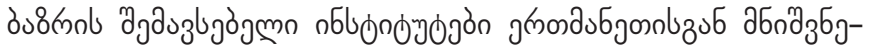

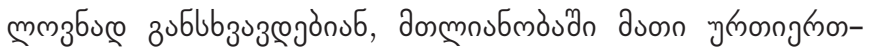

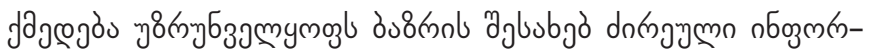

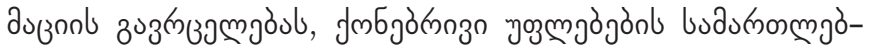

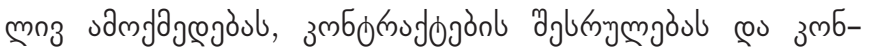

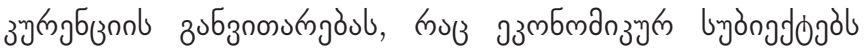

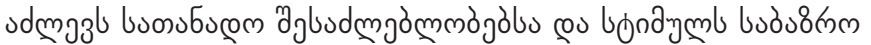

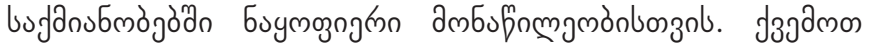

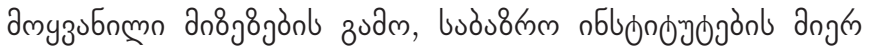

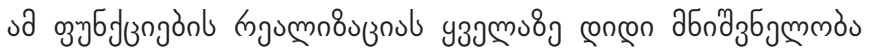
o (Acemoglu, Aghion, Zilibotti, 2002).

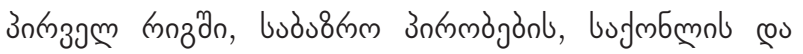

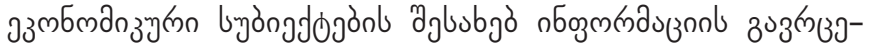

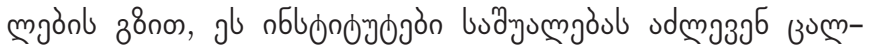

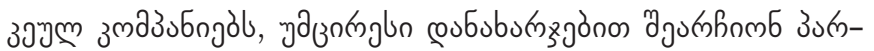

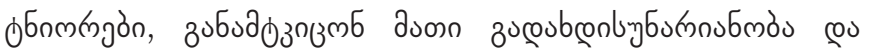

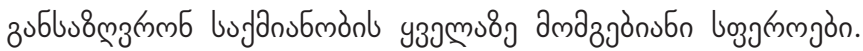

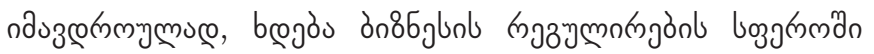

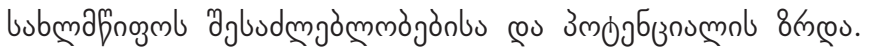

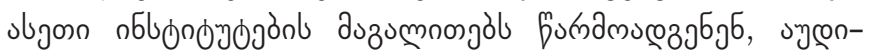

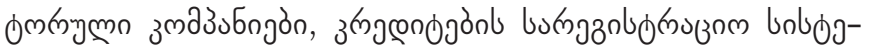

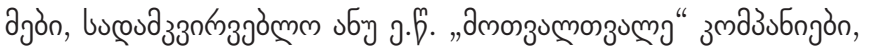

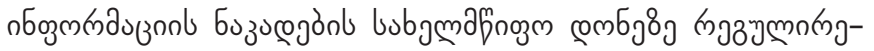

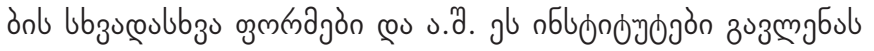

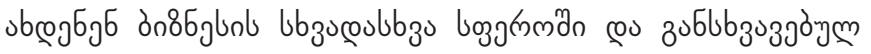

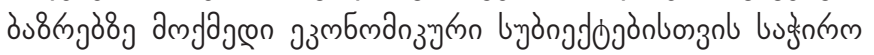

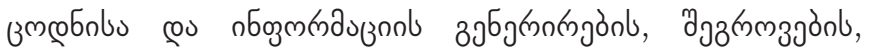

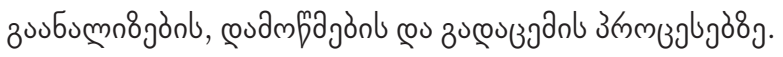

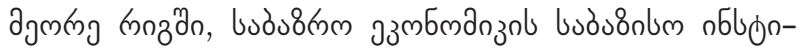

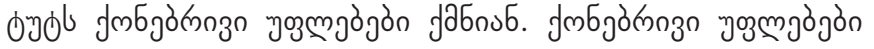

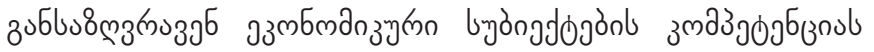

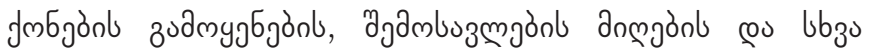

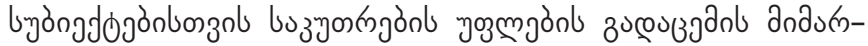

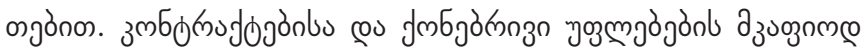

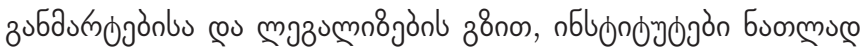
зงбbu8

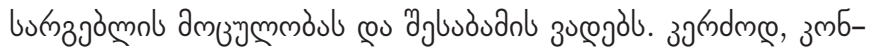

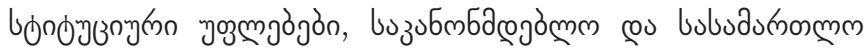

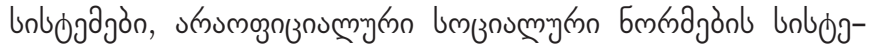

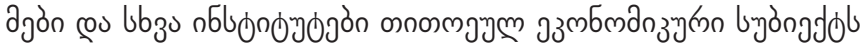

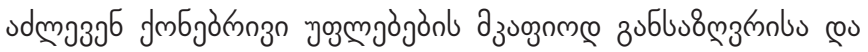

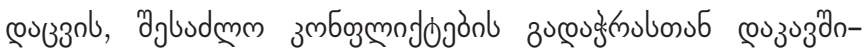

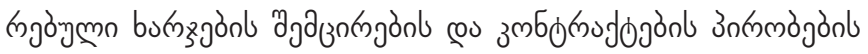

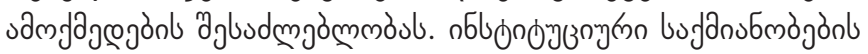

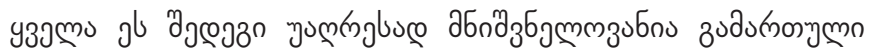

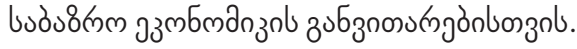

a

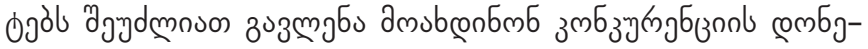

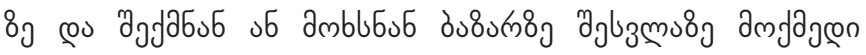

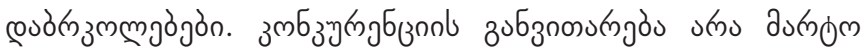

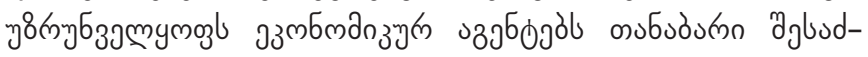

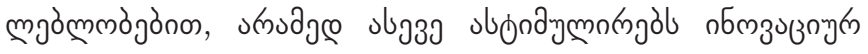

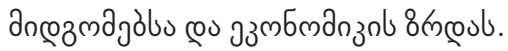

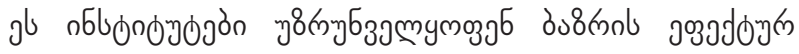

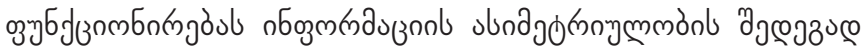

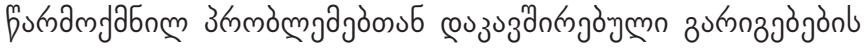

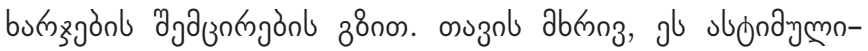
r

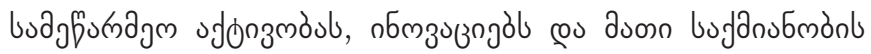
उ mmeñdonymmònl 8knoul (World Bank, 2001).

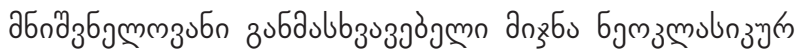

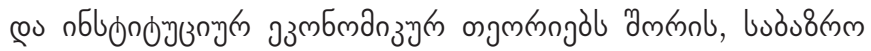

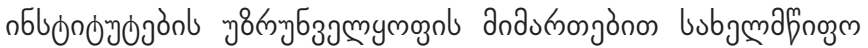

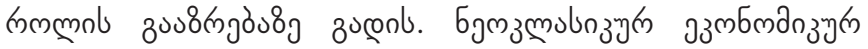

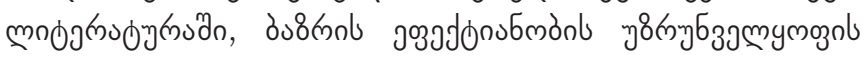

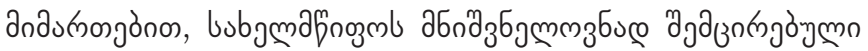

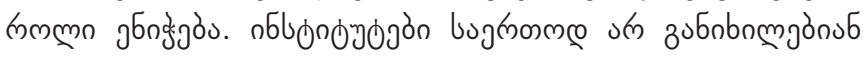

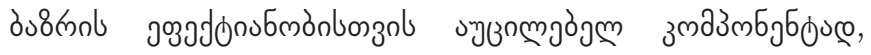

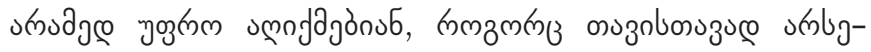

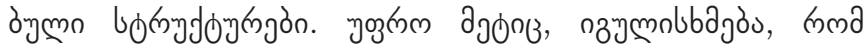

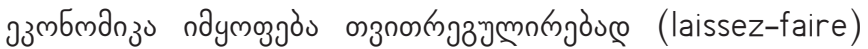

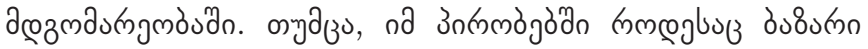
um skn

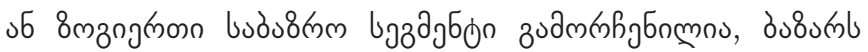

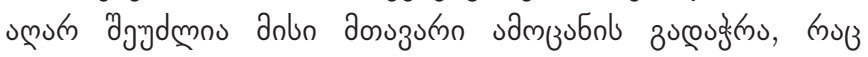

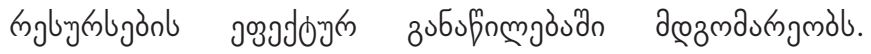

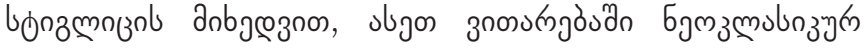

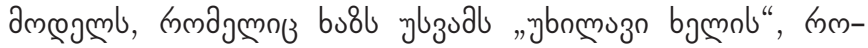

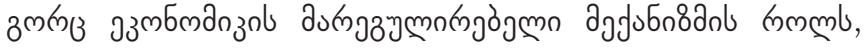

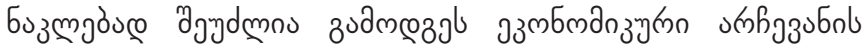

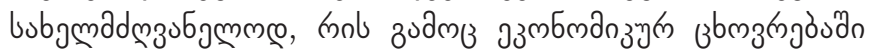




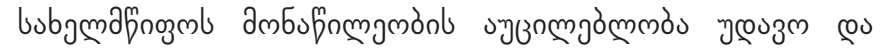

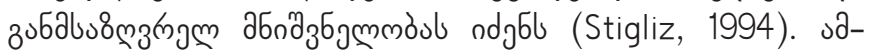

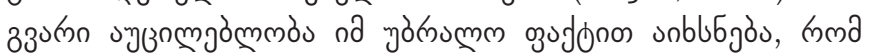

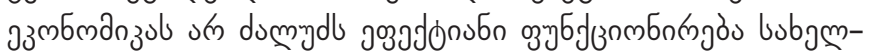

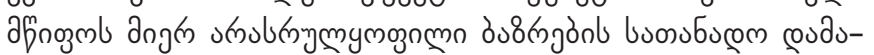

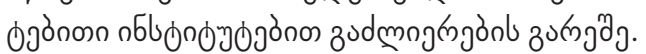

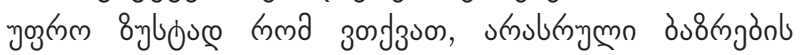

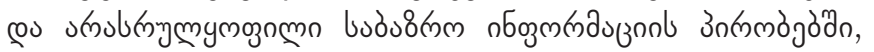

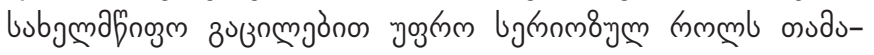

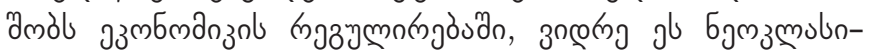
उचु

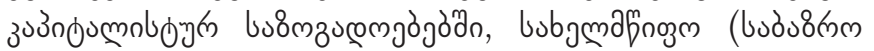

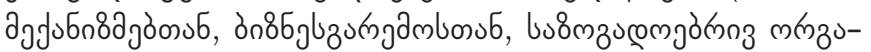

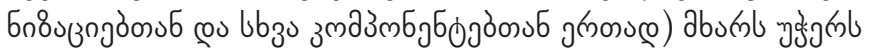

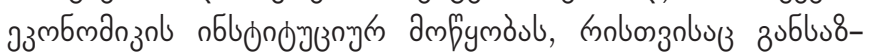

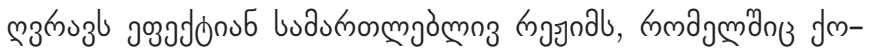

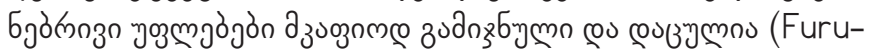
botn and Richter, 2003; North, 1990).

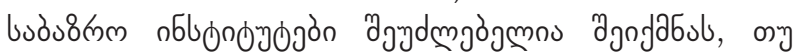

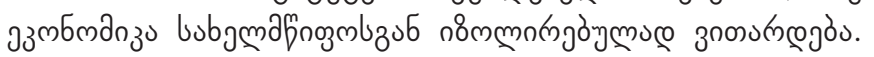

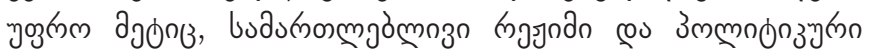

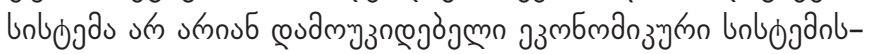

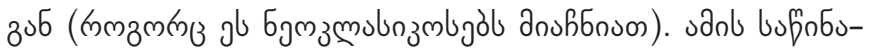

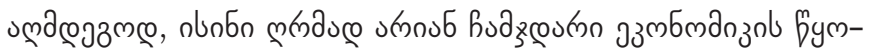

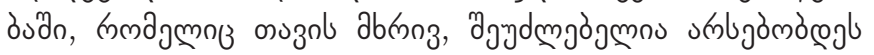

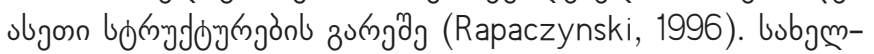

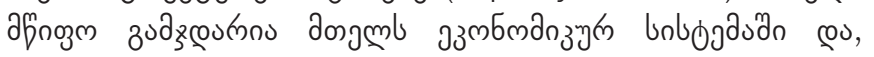

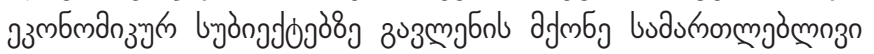

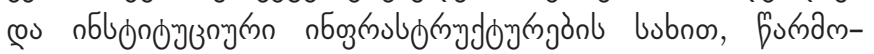

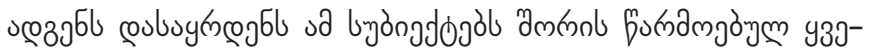

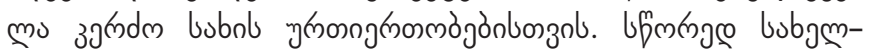

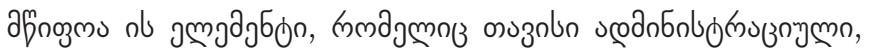

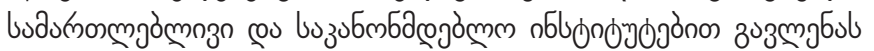

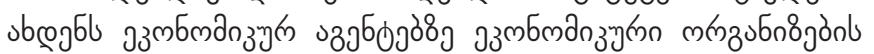

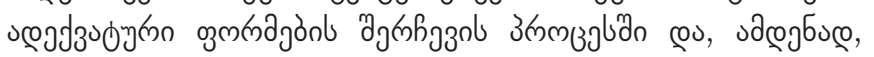
zublu8 po buouonlum dububnuongòmgall (Campbell and Lindberg, 1990).

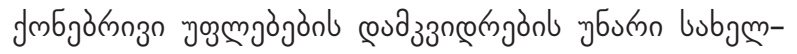

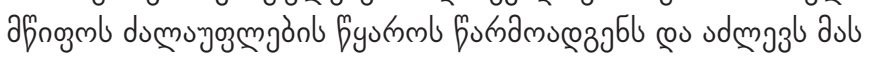

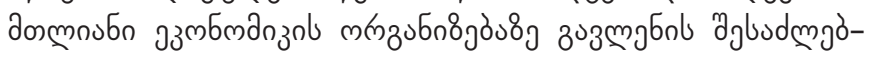
mmòul. uf

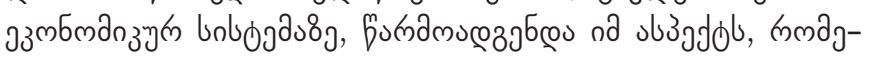

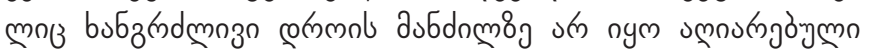

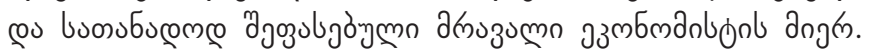

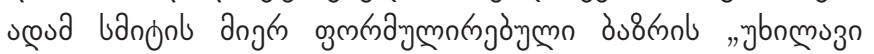

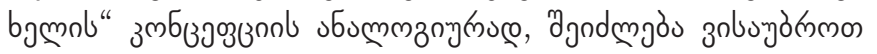

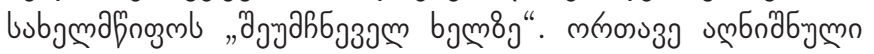

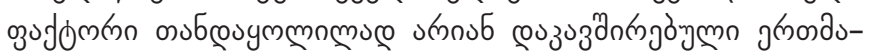

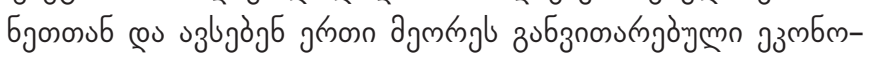

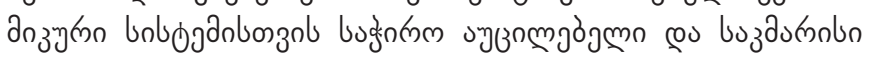

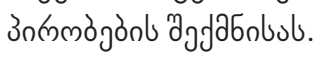

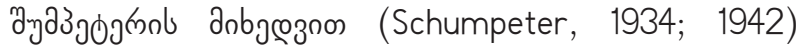

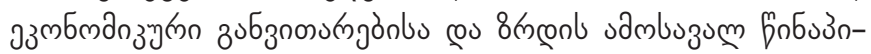

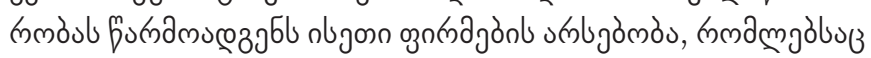

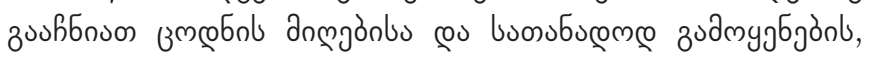

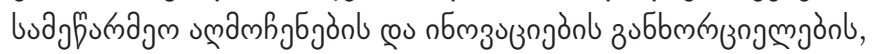

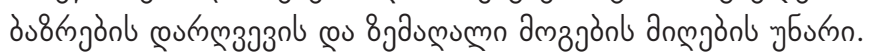

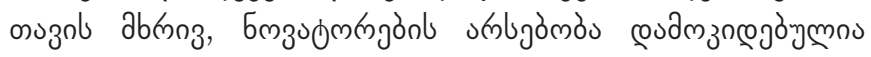

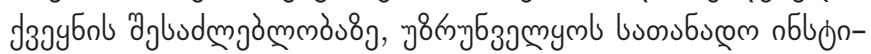

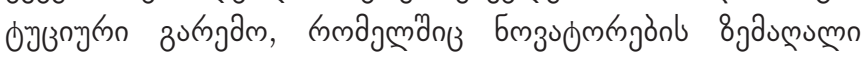

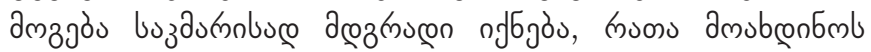

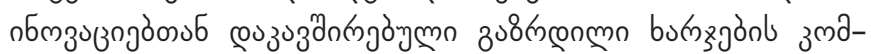

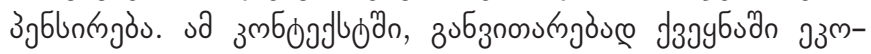

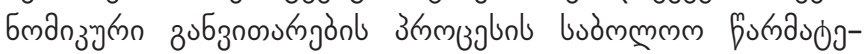

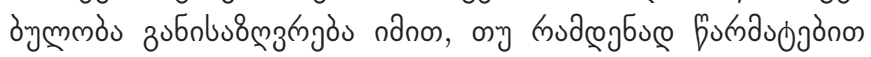

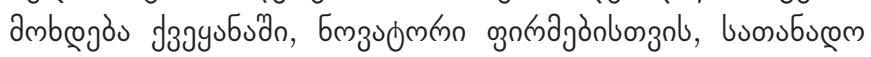

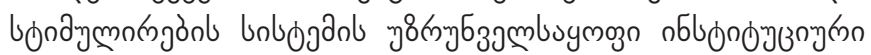

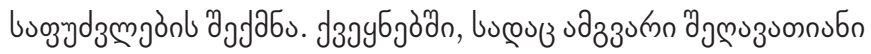

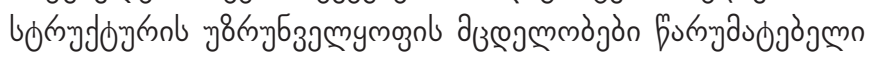

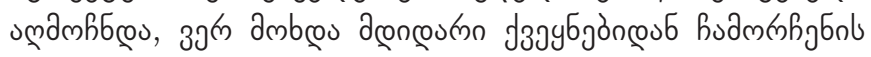

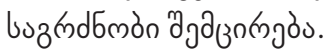

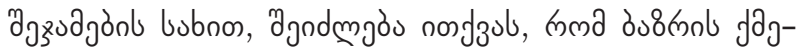

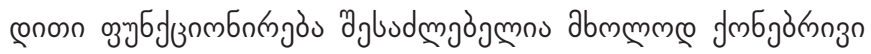

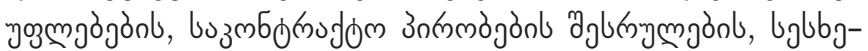

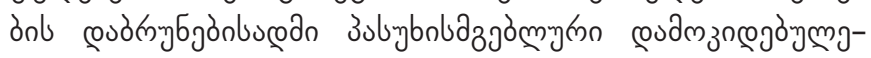

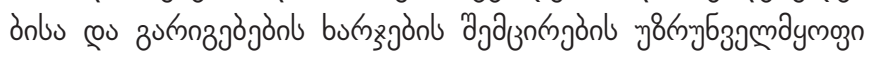

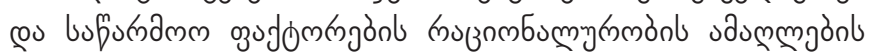

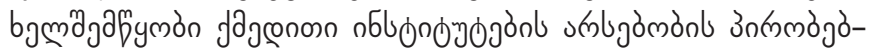

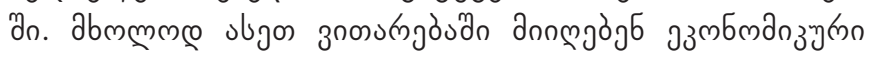

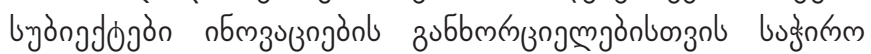

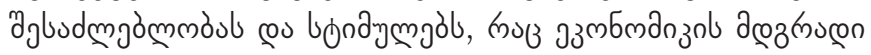

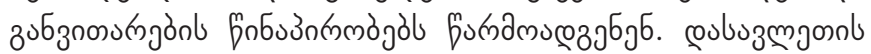

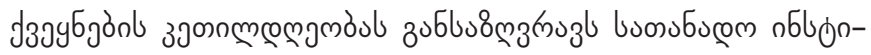

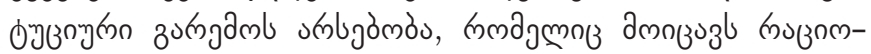

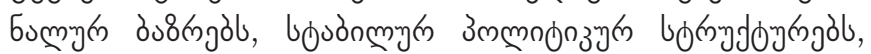

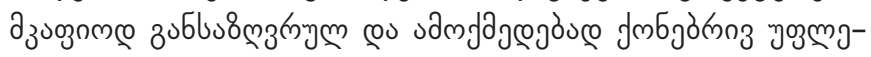

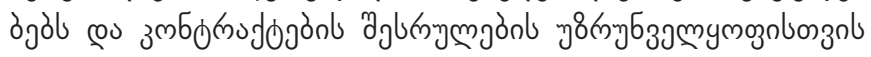

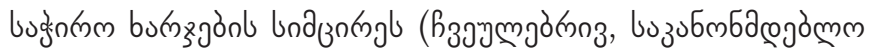

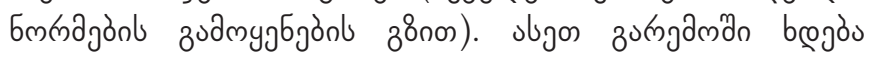

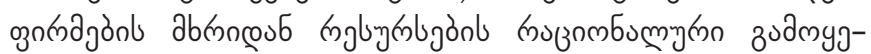
бjònl, ousdjm

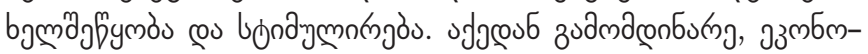

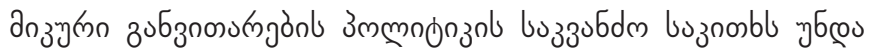

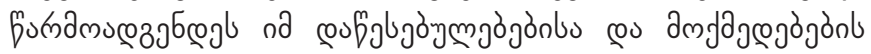

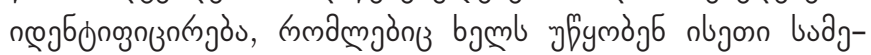

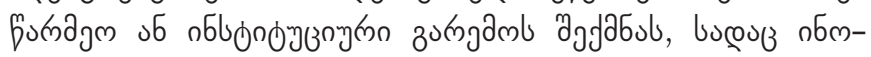

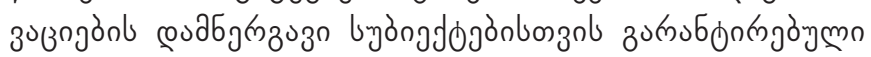

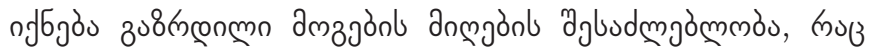

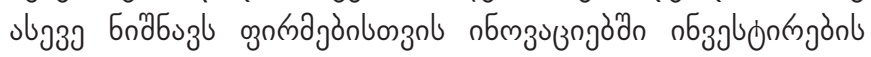

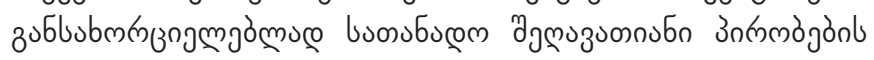
gjadabul. 


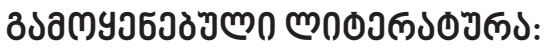

1. Acemoglu, D., P. Aghion, and F. Zilibotti (2002). „Distance to Frontier, Selection, and Economic Growth," NBER Working Paper No. 9066, July.

4. Campbell, L. J. and L. N. Lindberg (1990). „Property Rights and the Organization of Economic Activity by the State“, American Sociological Review, Vol. 55, Issue 5 (October): pp. 634-647.

5. Furubotn, G., E. and R. Richter (2003). Institutions and Economic Theory: The Contribution of the New Institutional Economics. Ann Arbor: The University of Michigan Press.

6. North, D. C. (1991). „Institutions“, The Journal of Economic Perspectives, Vol. 5, Issue 1 (Winter), pp.97-112.

7. North, D. C. (1990). Institutions, Institutional Change and Economic Performance. Cambridge: Cambridge University Press.

8. North, D. C. and R. P. Thomas (1973). The Rise of the Western World: A New Economic History. Cambridge University Press, Cambridge UK.

9. Rapaczynski, A. (1996). "The Role of the State and the Market in Establishing Property Rights", Journal of Economic Perspectives, Vol. 10., N2 (Spring): pp. 87-103.

10. Schumpeter, J. A. (1934). The Theory of Economic Development. Cambridge, MA: Harvard University Press.

11. Schumpeter, J. A. (1942). Capitalism, Socialism and Democracy. New York: Harpers and Row.

12. Stiglitz, J.E. (1994). Whither Socialism? Cambridge: Cambridge University Press.

13. Williamson, O. E. (1981 ). „The Economics of Organization: The Transaction Cost Approach“, American Journal of Sociology, Vol.87, Issue 3 (Nov), pp.548-577.

14. World Bank, The (2001). World Development Report 2002: Building Institutions for Markets. The World Bank, Washington DC. 


\section{ON THE ROLE OF MARKET INSTITUTIONS DURING ECONOMIC TRANSFORMATION PROCESS}

\section{GEORGE BERULAVA}

https://doi.org/10.35945/gb.2018.06.016

Doctor of Economic Sciences, Professor.

Ivane Javakhishvili Tbilisi State University

Paata Gugushvili Institute of Economics

Academician of the Georgian Academy of Economic Sciences, Georgia

george.berulava@gmail.com

KEYWORDS: ECONOMIC TRANSFORMATION PROCESS, MARKET INSTITUTIONS

ECONOMIC DEVELOPMENT, INNOVATIONS

\section{SUMMARY}

The paper considers the role market institution in the process of economic transformation. At present stage, the development of the efficient market system implies the creation of the incentive structure that will stimulate businesses to invest in innovation and production activities rather than in 'rent-seeking' behavior. Such an incentive structure, in turn, is a product of viable institutions that complement the mechanism

of market competition and that guarantee property rights, honour of contracts and payment of credits, lower barriers for market entry and exit, reduce transaction costs and facilitate increase of the effectiveness of the market of production factors. Only under such conditions economic agents receive the possibility and stimuli for innovation, thus creating preconditions for steady economic growth. 